August, 2015

"Earmarking and the political support of fat taxes"

Helmuth Cremer, Catarina Goulaõ, Kerstin Roeder 


\title{
Earmarking and the political support of fat taxes ${ }^{1}$
}

\author{
Helmuth Cremer ${ }^{2}$ \\ Toulouse School of Economics (GREMAQ, IDEI, IUF) \\ Catarina Goulão \\ Toulouse School of Economics (GREMAQ, INRA) \\ Kerstin Roeder \\ University of Augsburg
}

April 2015, revised August 2015

\footnotetext{
${ }^{1}$ We thank Pierre Pestieau and all participants of the EHEW in Toulouse for their helpful comments and suggestions.

${ }^{2}$ Financial support from the Chaire "Marché des risques et creation de valeur" of the FdR/SCOR is gratefully acknowledged.
} 


\begin{abstract}
A fat and a healthy good provide immediate gratification, and cause health costs or benefits in the long run, which are misperceived. Additionally, the fat good (healthy good) increases (decreases) health care costs by increasing (decreasing) the probability of suffering from a chronic disease in the future. Individuals differ in income and in their degree of misperceptions concerning the health effects of the consumption of fat and of healthy goods. The level of the fat tax is determined through majority voting. Individuals vote according to their misperceived utility function. Consequently, excessive fat consumption is not due to a self-control problem but due to information deficiencies or cognitive inability to process information. A fraction of the fat tax proceeds is "earmarked" to reduce health insurance premiums while the remaining fraction finances a subsidy on the healthy good. This earmarking rule is determined at a constitutional stage to maximize utilitarian or Rawlsian welfare, anticipating the induced political equilibrium. We show that the fat tax in the political equilibrium is always lower than the utilitarian fat tax. This is no longer necessarily true with a Rawlsian objective. The determination of the optimal earmarking rule is quite complex. Even in the utilitarian case, it is not just used to boost political support for the fat tax. Instead, it may involve a tradeoff between the fat tax and the healthy good subsidy.
\end{abstract}

Keywords: obesity, fat tax, misperception, voting, earmarking JEL Codes: I12, I18, D72 


\section{Introduction}

Worldwide obesity has more than doubled since the 1980s. In $2014,33 \%$ of the US and $23 \%$ of the European populations were obese. More than $70 \%$ of the US and UK population are overweight, and worldwide there are examples of similar patterns even among developing countries such as Argentina and Chile, or middle eastern countries such as Kuwait, Qatar, and Saudi Arabia. ${ }^{1}$ Because of such high prevalence rates, obesity is considered an epidemic despite being non-infectious.

The statistics on the prevalence of obesity are worrying because, along with tobacco consumption, overweight and obesity are risk factors of non-communicable chronic diseases (NCDs), which are presently the leading cause of deaths worldwide. Examples of NCDs are heart disease, stroke, diabetes, musculoskeletal disorders like osteoarthritis, and some sorts of cancer. According to the WHO, NCDs account for over $60 \%$ of the total deaths in the world, and half of these deaths occurred in low- and middle-income countries before age $70 .^{2}$

In addition to being a main factor in premature mortality, NCDs are also responsible for extended periods of morbidity and dependency. These impediments may dramatically reduce individuals' quality of life. Additionally, they lead to a significant increase in present health care costs worldwide even if an individual's full lifetime is accounted for. For example, Cawley and Meyerhoefer (2012) estimate obesity medical costs of 2,741 USD, which translates in $20 \%$ of the US health expenditure. Finkelstein et al. (2008) find that the severely obese white women have the highest full lifetime health care cost of 29,460 USD. $^{3}$

There are many factors put forward as reasons for the obesity spread (Cutler et al., 2003), but in the end it typically boils down to unbalanced caloric intakes compared to energy needs. Consequently, a policy intervention aiming at eradicating, or at least slowing down the epidemics has to affect diet and physical activity choices. Of course obesity also has a genetic component. However, genetics cannot explain the dramatic increase in obesity rates over the last three decades (Hill and Peters, 1998).

In this paper we analyze the political support of a "fat tax", imposed on fattening

\footnotetext{
${ }^{1}$ Overweight and obesity prevalence rates statistics available from the World Health Organization (WHO), http : //apps.who.int/gho/data/view.main.2461A?lang = en accessed on June 26, 2015.

${ }^{2}$ http://www.who.int/mediacentre/factsheets/fs355/en/, accessed on June 26, 2015.

${ }^{3}$ See as well Finkelstein et al., 2003; Finkelstein et al., 2011; Finkelstein and Yang, 2011; Thorpe et al., 2004; Wang et al., 2008; and Wang et al., 2011; among others.
} 
food and beverages, combined with a subsidy on healthy goods. These are amongst the policy instruments with the potential to slow down the obesity epidemics. We also examine how the government can affect this political support by "earmarking" the tax revenue.

In reality fat taxes exist, but remain relatively rare and their level is not very significant. A fat tax was first introduced in Denmark in October 2011, yet withdrawn one year later. Currently, fat taxes are being discussed in several countries such as the UK or France. ${ }^{4}$ In 2009, 33 states in the USA taxed soft drinks, and more recently France, Norway and Mexico have imposed similar taxes (Brownell et al., 2009). The relative scarcity of fat taxes in practice suggests that such a policy may not be sufficiently attractive from a political perspective.

Intuitively, one can expect the following factors to affect the political support of fat taxes. First, they are likely to be regressive. Low-income individuals consume more fattening food and beverages, in particular, because they often misperceive the effects of diet and physical activity in the short and the long run (see Vereecken and Maes, 2010; and Bauman et al., 2012; among others). ${ }^{5}$ Consequently, the median income individual bears a larger than average burden of the tax; this may lead to a lower than optimal level of the fat tax in a voting equilibrium. Second, irrespective of distributional considerations, misperception in itself may imply that individuals underestimate the benefits of the tax which undermines its political support. Third, in countries with SHI (Social Health Insurance) or NHS (National Health System), individuals may not properly account for the social health care costs caused by their unhealthy lifestyle.

In this paper we study the political determination of fat taxes in a simple setting. We show that the equilibrium is typically not optimal, neither from a utilitarian nor from a Rawlsian perspective. More precisely, the equilibrium level of the fat tax is always lower than the utilitarian optimum, but it may be lower or higher than the Rawlsian solution. However, this is just a prelude to the main question we ask, namely if and how the government can affect the political support for fat taxes through "an accompanying" policy of earmarking to bring the voting solution closer to the optimum.

\footnotetext{
${ }^{4}$ See "UK could introduce a fat tax, says David Cameron", theguardian.com, Tuesday 4 October 2011 13.50 BST, http ://www.theguardian.com/politics/2011/oct/04/uk-obesity-tax-david-cameron, accessed on June 26, 2014 and IGF (2008); Bonnet et al. (2009); and Allais et al. (2010).

${ }^{5}$ However the regressive nature of a fat tax may be compensated by the health benefits it induces through the improvement of diet.
} 
We consider an economy wherein agents differ in their income and in their degree of misperceptions concerning the health effects of fat and of healthy goods. Consumption of the fat good gives rise to immediate satisfaction but also causes adverse health effects, which are misperceived. In turn, the consumption of the healthy good has beneficial health effects, which are misperceived as well. ${ }^{6}$

The fat good consumption increases the probability of suffering from NCDs in the future. We assume that all sick individuals are treated within a national health system, which is financed by a uniform capitation fee. Therefore, individuals' fat consumption imposes higher taxes on all due to the need to balance the budget of the national health system. In such a setup, we investigate the role of a fat tax aiming to correct: (i) individuals' misperceptions concerning health effects, and (ii) the negative effect of an increase in the health care costs. Since a fat tax constitutes a new source of financing the capitation fee can be cut down. Additionally, the capitation fee can be reduced further because health care expenses decrease with a decrease in fat consumption.

We consider the possibility that the fat tax proceeds are used either to contribute to the financing of the health care system or to subsidize the consumption of some healthy good or activity (see Cremer et al., 2010; and Goulão and Pérez-Barahona, 2014). In other words, the proceeds of the fat tax are earmarked so that some fraction is used to finance health care while the remaining part finances a subsidy on the healthy good consumption (for the later see Yaniv et al., 2009). ${ }^{7}$ This earmarking rule is determined by a welfare maximizing (utilitarian or Rawlsian) government at a constitutional stage which precedes the vote over the fat tax. It is by now well known that earmarking is at best useless in a purely normative setting. However, it has interesting effects when all or part of the policy instruments are determined by a political process. In particular, we show how earmarking of the fat tax revenues can be used to boost the political support of a more welfare enhancing fat tax. The voting equilibrium tax rate increases as the fraction of tax revenues devoted to the financing of health insurance increases.

\footnotetext{
${ }^{6}$ There seems to exist a consensus that the population is generally poorly informed about the attributes of a healthy diet (Vereecken and Maes, 2010; Grimes et al., 2009; and Schwartz et Byrd-Bredbenner, 2005 ) and there is evidence that greater nutritional knowledge is related to better nutrition (Pollard et al., 2010; and Vereecken and Maes, 2010).

${ }^{7}$ This is pretty much in line with the allocation of fat tax suggested by Leicester and Windmeijer (2004, page 10) who argue that "Funds from a 'fat tax' could be used to finance healthy eating advice in schools or on television, for the NHS, etc. Alternatively, the income could be used to reduce other taxes or provide subsidies on healthy foods or exercise equipment."
} 
Consequently, one might expect that a utilitarian government would choose to devote a larger share of tax revenue to health expenses than otherwise optimal. We show that this is not necessarily true because an increase in this share tends to decrease the subsidy on the healthy good (which is also too low at the utilitarian solution). Similar effects are at work in the Rawlsian case with the added complexity that the voting equilibrium tax level may then actually exceed the optimal level.

Our paper contributes to a growing literature on sin taxes. O'Donoghue and Rabin (2003; 2006) study precisely the relevance of sin taxes in view of correcting individuals misperceptions. They conclude that they may even be Pareto improving since they help people with self-control problems without imposing losses to fully rational people.

Some political economy aspects of sin taxes have been studied by Haavio and Kotakorpi (2011). They show how voting over a sin tax can work as a commitment device for individuals with self-control problems. Our approach departs from theirs in two main directions. First, we consider that individuals vote according to their misperceived utility function. In other words, we assume that excessive fat consumption is not due to a self-control problem. Instead, an individual's misperception of health effects is due to information deficiencies or cognitive inability to process information. Moreover, we suppose that higher income individuals have lower misperception levels. Second, we consider the impact of fat consumption on the costs of the health care system. This feature together with the possibility of earmarking the funds of the fat tax to subsidize a healthy activity make the political problem more complex but generates interesting equilibria.

The remainder of the paper is organized as follows. Section 2 describes our economic environment. Then, from a normative perspective, the optimal fat tax and budgetary rule are analyzed in Section 3. The political fat tax is determined in Section 4. The constitutional stage is analyzed in Section 5 and Section 6 provides some concluding remarks.

\section{The economic environment}

The economy consists of individuals who differ in income $y_{i}$ which is distributed on the support $\left[y_{\ell}, y_{h}\right] \in \mathbb{R}^{+}$according to the cumulative distribution function $F(y)$. Population size is normalized at one. Individual's utility depends on the consumption of an 
unhealthy, high fat or sugar good $\varphi_{i}$, (e.g., potato chips or sodas), a healthy good $x_{i}$ (e.g., vegetables), and a numeraire good $c_{i}$ (e.g., leisure). To simplify the exposition we will often refer to the unhealthy commodity as "fat good", but our analysis applies just as well to high sugar products. All producers' prices are normalized at one. Fat goods are taxed at a rate $\theta \geq 0$ and healthy goods are subsidized at a rate $s \geq 0$.

The satisfaction (and health benefits) individuals obtain from the fat good is reflected by $v\left(\varphi_{i}\right)$ with $v^{\prime}\left(\varphi_{i}\right)>0$ and $v^{\prime \prime}\left(\varphi_{i}\right)<0$. In the long run, however, higher consumption of fat causes overweigh or obesity and comes with health problems. These negative effects of fat good consumption are captured by the harm function $h\left(\varphi_{i}\right)$ with $h^{\prime}\left(\varphi_{i}\right)>0$ and $h^{\prime \prime}\left(\varphi_{i}\right)>0$. In the short run, individuals may ignore part of these negative long-run effects. Their perceived harm function is given by $\beta_{i} h\left(\varphi_{i}\right)$ where $\beta_{i} \in[0,1]$. We assume that $\beta_{i} \equiv \beta\left(y_{i}\right)$ decreases with income, i.e., $\beta^{\prime}\left(y_{i}\right)>0$. The empirical support for this correlation is broad; see, e.g., Devaux et al., (2011), and McLaren (2011) for evidence on the negative association of socio-economic status/education and obesity/nutritional knowledge.

The healthy good provides a utility of $b\left(x_{i}\right)$ with $b^{\prime}\left(x_{i}\right)>0$ and $b^{\prime \prime}\left(x_{i}\right)<0$. In line with the empirical evidence these benefits are also misperceived by some agents implying $\delta_{i} b\left(x_{i}\right)$, where $\delta_{i} \in[0,1]$. Once again the degree of misperception depends on income, $\delta_{i} \equiv \delta\left(y_{i}\right)$, and is higher the lower individuals' income: $\delta^{\prime}\left(y_{i}\right)>0$. Finally, utility is linear in the numeraire good.

Consumption of fat and healthy goods determines the probability that an individual will develop a chronic disease, $\pi\left(\varphi_{i}, x_{i}\right)$. This probability is increasing in the fat good consumption and decreasing in the healthy good consumption. For simplicity we assume $\partial^{2} \pi / \partial \varphi_{i} \partial x_{i}=0$. The government supplies "free" health care to those suffering from a chronic disease. The health care scheme provides universal and full coverage and is financed by capitation fees $\tau$. The cost of treating patients is normalized to one.

In sum, individual $i$ 's perceived utility, $\widehat{U}_{i}$, is given by

$$
\widehat{U}_{i}=c_{i}+\delta_{i} b\left(x_{i}\right)+v\left(\varphi_{i}\right)-\beta_{i} h\left(\varphi_{i}\right),
$$

while the individual's true utility, $U_{i}$, amounts to

$$
U_{i}=c_{i}+b\left(x_{i}\right)+v\left(\varphi_{i}\right)-h\left(\varphi_{i}\right)
$$

Since all patients are treated and cured $\pi\left(\varphi_{i}, x_{i}\right)$ does not directly appear in individuals' 
utilities, but it of course affects utility indirectly through the taxes levied to finance health care expenditure.

\subsection{Individual optimization problem}

Individuals choose their consumption of the fat and the healthy goods by maximizing perceived utility specified by equation (1), subject to their budget constraint, i.e.

$$
\begin{aligned}
\max _{c_{i}, x_{i}, \varphi_{i}} & \widehat{U}_{i}=c_{i}+\delta_{i} b\left(x_{i}\right)+v\left(\varphi_{i}\right)-\beta_{i} h\left(\varphi_{i}\right) \\
\text { s.t. } & c_{i}=y_{i}-\tau-(1+\theta) \varphi_{i}-(1-s) x_{i} .
\end{aligned}
$$

The first order conditions (FOCs) are given by

$$
\begin{aligned}
& \frac{\partial \widehat{U}_{i}}{\partial x_{i}}=-(1-s)+\delta_{i} b^{\prime}\left(x_{i}\right)=0, \\
& \frac{\partial \widehat{U}_{i}}{\partial \varphi_{i}}=-(1+\theta)+v^{\prime}\left(\varphi_{i}\right)-\beta_{i} h^{\prime}\left(\varphi_{i}\right)=0 .
\end{aligned}
$$

These equations determine the demand functions $x_{i}^{*} \equiv x\left(s, \delta_{i}\right)$ and $\varphi_{i}^{*} \equiv \varphi\left(\theta, \beta_{i}\right)$. The comparative statics with respect to the tax and subsidy, and with respect to the misperception parameters are as follows:

$$
\begin{aligned}
\frac{\partial x_{i}^{*}}{\partial s} & =-\frac{1}{\delta_{i} b^{\prime \prime}\left(x_{i}\right)}>0, \\
\frac{\partial x_{i}^{*}}{\partial \delta_{i}} & =-\frac{b^{\prime}\left(x_{i}\right)}{\delta_{i} b^{\prime \prime}\left(x_{i}\right)}>0, \\
\frac{\partial \varphi_{i}^{*}}{\partial \theta} & =\frac{1}{v^{\prime \prime}\left(\varphi_{i}\right)-\beta_{i} h^{\prime \prime}\left(\varphi_{i}\right)}<0, \\
\frac{\partial \varphi_{i}^{*}}{\partial \beta_{i}} & =-\frac{-h^{\prime}\left(\varphi_{i}\right)}{v^{\prime \prime}\left(\varphi_{i}\right)-\beta_{i} h^{\prime \prime}\left(\varphi_{i}\right)}<0 .
\end{aligned}
$$

Not surprisingly, a higher subsidy on healthy goods and a lower misperception of their health benefits increase their consumption. Similarly, a higher tax on fat goods and a lower misperception of their negative health effects decrease an individual's fat good consumption. Since low-income individuals suffer from a higher misperception they tend to consume more fat and less healthy goods than individuals with higher income and lower misperception.

\subsection{Equilibrium}

In an equilibrium, public budgets need to be balanced. Health care costs are given by

$$
\int_{y_{\ell}}^{y_{h}} \pi[\varphi(\theta, \beta(y)), x(s, \delta(y))] \mathrm{d} F(y) \equiv \bar{\pi}(\theta, s) .
$$


They are financed by capitation fees $\tau$ and the revenue raised from the $\operatorname{tax} \theta$ on the fat good. The fat tax revenue is given by

$$
\int_{y_{\ell}}^{y_{h}} \theta \varphi(\theta, \beta(y)) \mathrm{d} F(y) \equiv \theta \bar{\varphi}(\theta) .
$$

A share $\alpha \leq 1$ of fat tax revenue is earmarked to finance the health care system while the remaining share $1-\alpha$ is used to subsidize the healthy good consumption. The government balances both the health care budget and the budget for the healthy good subsidies. These two constraints determine $\tau$ and $s$ as functions of $\theta$ and $\alpha$. Specifically, we have

$$
\begin{aligned}
(1-\alpha) \theta \bar{\varphi}(\theta) & =s \bar{x}(s) \quad \Rightarrow \quad s(\theta, \alpha), \\
\tau+\alpha \theta \bar{\varphi}(\theta) & =\bar{\pi}(\theta, s(\theta, \alpha)) \quad \Rightarrow \quad \tau(\theta, \alpha),
\end{aligned}
$$

where $\bar{x}(s) \equiv \int_{y_{\ell}}^{y_{h}} x(s, \delta(y)) \mathrm{d} F(y)$ is the average consumption level of the healthy good.

Note that we do not restrict $\alpha$ to be positive. To understand this, observe that equation (11) implies

$$
1-\alpha=\frac{s \bar{x}(s)}{\theta \bar{\varphi}(\theta)} .
$$

In words, $(1-\alpha)$ measures the ratio between the budgetary cost of the subsidy on the healthy good and the tax revenue collected on the fat good. With $s \geq 0$ and $\theta \geq 0$, we have $1-\alpha>0$ so that $\alpha>1$ is not possible. However, there is no good reason to rule out the case where $s \bar{x}(s)>\theta \bar{\varphi}(\theta)$ so that $\alpha<0$. In this case, the remaining part of the subsidy on the healthy good is financed by the capitation fee, as shown by (12).

Assuming a positive fat tax, the effect of $\theta$ on $\tau(\theta, \alpha)$ and $s(\theta, \alpha)$ is given by

$$
\begin{aligned}
& \frac{\partial s}{\partial \theta}=\frac{(1-\alpha) \bar{\varphi}\left(1-\varepsilon_{\bar{\varphi}, \theta}\right)}{\bar{x}\left(1+\varepsilon_{\bar{x}, s}\right)}>0, \\
& \frac{\partial \tau}{\partial \theta}=\bar{\pi}_{\theta}-\alpha \bar{\varphi}\left(1-\varepsilon_{\bar{\varphi}, \theta}\right)<0,
\end{aligned}
$$

where $\varepsilon_{\bar{\varphi}, \theta} \equiv-(\partial \bar{\varphi} / \partial \theta) \theta / \bar{\varphi}$ and $\varepsilon_{\bar{x}, s} \equiv(\partial \bar{x} / \partial s) s / \bar{x}$ are the elasticities of society's fat and healthy good consumption with respect to the fat tax and the subsidy respectively and $\bar{\pi}_{\theta} \equiv \partial \bar{\pi} / \partial \theta+(\partial \bar{\pi} / \partial s)(\partial s / \partial \theta)<0$. Note that a higher taxation of fat goods reduces the average probability of chronic disease and thus health care costs both directly by reducing $\varphi$ and indirectly by increasing $x$. In accordance with the empirical estimations of Allais et al.(2010); Beatty and La France (2005); Harding and Lovenheim (2013); and Huang and Lin (2000) among others, we assume that $\varepsilon_{\bar{\varphi}, \theta} \leq 1$. Consequently, equation 
(14) is positive and equation (15) is negative. In words, capitation fees decrease with the fat tax rate, whereas the subsidy on the healthy good increases with it. The intuition behind it is straightforward. If a $1 \%$ increase in the fat tax leads to a decrease in the aggregate fat good consumption by less than $1 \%$, a positive revenue from taxation is generated. This revenue can be used to reduce capitation fees and increase the subsidy on healthy activities.

The comparative static effects with respect to $\alpha$ are given by

$$
\begin{aligned}
& \frac{\partial s}{\partial \alpha}=\frac{-\theta \bar{\varphi}}{\bar{x}\left(1+\varepsilon_{\bar{x}, s}\right)}<0 . \\
& \frac{\partial \tau}{\partial \alpha}=\bar{\pi}_{\alpha}-\theta \bar{\varphi} \lessgtr 0,
\end{aligned}
$$

where $\bar{\pi}_{\alpha} \equiv(\partial \bar{\pi} / \partial s)(\partial s / \partial \alpha)>0$. The more of fat tax revenue is devoted to the health care scheme the lower are the subsidies on healthy goods. However, the impact of $\alpha$ on $\tau$ is ambiguous. The direct effect, measured by the second term in (17), is of course negative, because a larger share of the fat tax proceeds is devoted to the financing of the health insurance scheme. But there is also an indirect effect which goes in the opposite direction. This is because the decrease in subsidies on the healthy good will tend to increase health care cost.

For future reference, it is useful to express type- $i$ agents' perceived and true utility level by their indirect utility functions. Substituting $s(\theta, \alpha)$ and $\tau(\theta, \alpha)$, as defined by equations (11) and (12), into the perceived and true utility functions, (1) and (2), yields

$$
\begin{aligned}
\widehat{V}_{i}(\theta, \alpha)= & y_{i}-\tau(\theta, \alpha)-(1+\theta) \varphi_{i}(\theta)-(1-s(\theta, \alpha)) x_{i}(s(\theta, \alpha))+\delta_{i} b\left(x_{i}(s(\theta, \alpha))\right) \\
& +v\left(\varphi_{i}(\theta)\right)-\beta_{i} h\left(\varphi_{i}(\theta)\right), \\
V_{i}(\theta, \alpha)= & y_{i}-\tau(\theta, \alpha)-(1+\theta) \varphi_{i}(\theta)-(1-s(\theta, \alpha)) x_{i}(s(\theta, \alpha))+b\left(x_{i}(s(\theta, \alpha))\right) \\
& +v\left(\varphi_{i}(\theta)\right)-h\left(\varphi_{i}(\theta)\right) .
\end{aligned}
$$

Before analyzing the voting on the fat tax, we determine the socially optimal fat tax and budgetary rule, which provide a benchmark against which the properties of the political outcome can be assessed.

\section{Normative benchmark}

Since the public health care system is a prime ingredient of our setup, a "pure" laissezfaire $(L F)$ solution is ruled out by definition. The situation which comes closest to a 
conventional $L F$ corresponds to the case where $\theta=0$ which (through the earmarking rule) also implies $s=0$. It is plain that this solution is not efficient. To be more precise there are two sources of inefficiency. The first one is the individuals' misperception of the long-term effects of the fat and healthy good consumption. In addition, with a continuum of individuals health care costs are formally like an "atmosphere externality". Any given individual neglects the impact of his fat and healthy consumption on $\bar{\pi}$. Interestingly, this externality occurs even when aggregate health damages are correctly perceived by individuals. In other words, even when individuals are aware of equation (10) their consumption decisions will neglect its impact on health care cost.

We also know from standard textbook theory that in a world of perfect information these inefficiencies could be addressed through a system of individualized Pigouvian taxes (on $\varphi$ ) and subsidies (on $x$ ). We assume that such individualized taxes are not feasible, for instance because of asymmetries of information. Instead, as shown in the previous section, we restrict the government's instruments to linear taxes and subsidies with the same rate applied to all individuals.

Within this context we first examine the utilitarian solution. It is constrained by the linear instruments $(\theta, s)$, but they are both under the control of the welfare maximizing government. Note that with quasi-linear preferences, redistributive considerations do not matter for the utilitarian government. Yet, a common concern with fat taxes is their regressive nature; see, Allais et al. (2010); Frazão et al. (2007); Leicester and Windmeijer (2004); and Powell and Chaloupka (2009); among others. ${ }^{8}$ To address the redistributive nature of a fat tax we consider as well a Rawlsian government.

These solutions represent an interesting benchmark against which the political outcome considered in the subsequent sections can be assessed. This is the reason why we optimize with respect to $\theta$ and $\alpha$, the variable used in our political model, rather than with respect to $\theta$ and $s$. In a normative setting the change of variable is of no relevance. Using $\theta$ and $s$ as decision variable would effectively be simpler and more intuitive. In the utilitarian case this amounts to determining a Pigouvian tax and subsidy and the $\alpha$ is then determined purely mechanically from equation (13); see Appendix A.1.

\footnotetext{
${ }^{8}$ As noted by Katakorpi (2008), if low-income individuals have more elastic demand than high-income individuals a fat tax may even be progressive.
} 


\subsection{Utilitarian welfare function}

The utilitarian social planner chooses the fat tax and budgetary rule by maximizing the sum over individual's true net benefits of the fat and healthy good consumption as given by equation (19)

$$
\max _{\theta, \alpha} W^{U}(\theta, \alpha)=\int_{y_{\ell}}^{y_{h}} V_{i}(\theta, \alpha) \mathrm{d} F(y) .
$$

The FOC of (20) with respect to $\theta$ and $\alpha$ are given by

$$
\begin{aligned}
\frac{\partial W^{U}}{\partial \theta}= & -\frac{\partial \tau}{\partial \theta}-\bar{\varphi}-\int_{y_{\ell}}^{y_{h}}\left[(1+\theta)-\frac{\partial v_{i}}{\partial \varphi_{i}^{*}}+\frac{\partial h_{i}}{\partial \varphi_{i}^{*}}\right] \frac{\partial \varphi_{i}^{*}}{\partial \theta} \mathrm{d} F(y) \\
& +\frac{\partial s}{\partial \theta} \bar{x}-\int_{y_{\ell}}^{y_{h}}\left[(1-s)-\frac{\partial b_{i}}{\partial x_{i}^{*}}\right] \frac{\partial x_{i}^{*}}{\partial s} \frac{\partial s}{\partial \theta} \mathrm{d} F(y), \\
\frac{\partial W^{U}}{\partial \alpha}= & -\frac{\partial \tau}{\partial \alpha}+\frac{\partial s}{\partial \alpha} \bar{x}-\int_{y_{\ell}}^{y_{h}}\left[(1-s)-\frac{\partial b_{i}}{\partial x_{i}^{*}}\right] \frac{\partial x_{i}^{*}}{\partial s} \frac{\partial s}{\partial \alpha} \mathrm{d} F(y) .
\end{aligned}
$$

Using equations (4) and (5), these expressions reduce to

$$
\begin{aligned}
& \frac{\partial W^{U}}{\partial \theta}=-\frac{\partial \tau}{\partial \theta}-\bar{\varphi}+\frac{\partial s}{\partial \theta} \bar{x}+\frac{1}{\theta} \Omega(\beta, \delta)=0 \\
& \frac{\partial W^{U}}{\partial \alpha}=-\frac{\partial \tau}{\partial \alpha}+\frac{\partial s}{\partial \alpha} \bar{x}+\int_{y_{\ell}}^{y_{h}}\left(1-\delta_{i}\right) \frac{\partial b_{i}}{\partial x_{i}^{*}} \frac{\partial x_{i}^{*}}{\partial s} \frac{\partial s}{\partial \alpha} \mathrm{d} F(y),
\end{aligned}
$$

where we define

$$
\Omega(\beta, \delta) \equiv \int_{y_{\ell}}^{y_{h}}\left[\left(1-\beta_{i}\right) \varepsilon_{h_{i}, \theta} h\left(\varphi_{i}^{*}\right)+\left(1-\delta_{i}\right) \varepsilon_{b_{i}, \theta} b\left(x_{i}^{*}\right)\right] \mathrm{d} F(y)>0,
$$

to simplify notation. The elasticities of the fat tax on the harm of fat good consumption and on the benefit of the healthy good consumption are defined as

$$
\varepsilon_{h_{i}, \theta} \equiv-\frac{\partial h_{i}}{\partial \varphi_{i}^{*}} \frac{\partial \varphi_{i}^{*}}{\partial \theta} \frac{\theta}{h_{i}}, \quad \text { and } \quad \varepsilon_{b_{i}, \theta} \equiv \frac{\partial b_{i}}{\partial x_{i}^{*}} \frac{\partial x_{i}^{*}}{\partial s} \frac{\partial s}{\partial \theta} \frac{\theta}{b_{i}} .
$$

Expressions (23) and (24) represent the tradeoffs which determine the optimal levels of $\theta$ and $\alpha$. The first term of (23) reflects the marginal benefits of a higher fat tax revenue via the induced reduction in health care premiums. The second term measures the direct (average) utility loss associated with the price increase brought about by the fat tax. Similarly, the third term represents the (direct) utility gain associated with the induced subsidy on the healthy good. The last (Pigouvian) term represents the correction for the individuals' misperception of the health impact of the fat and healthy good. Setting the derivative equal to zero and rearranging yields

$$
\theta^{U}=\frac{\bar{\pi} \varepsilon_{\bar{\pi}, \theta}+\Omega(\beta, \delta)}{\bar{\varphi}\left[1-\left(1-\varepsilon_{\bar{\varphi}, \theta}\right)\left(\alpha+(1-\alpha) \frac{1}{1+\varepsilon_{\bar{x}, s}}\right)\right]}>0
$$


where $\varepsilon_{\bar{\pi}, \theta} \equiv-\bar{\pi}_{\theta} \theta / \bar{\pi}>0$. In words, the utilitarian level of the fat tax is positive. This is true even when there is no misperception of the health effects so that $\Omega(\beta, \delta)=0$. In that case, any given individual still neglects the impact of his fat and healthy good consumption on $\bar{\pi}$ and this calls for a corrective tax.

Turning to (24), the first term measures the impact of $\alpha$ on health care costs and thus on the capitation fee. Its sign is ambiguous; see equation (17). The second and third term are both negative. An increase in $\alpha$ lowers $s$ which has a negative direct effect on utility and also lowers the corrective (Pigouvian) effect of $s$. When $\tau$ increases with $\alpha$ it is clear that increasing $\alpha$ has only negative effects: it decreases $s$ and increases $\tau$. If instead $\tau$ decreases with $\alpha$, the solution trades off the negative effect of the decrease in $s$ against the positive effect of a decrease in $\tau$.

For the rest, equation (24) is not very informative about the sign of $\alpha$. This issue can, however, be addressed in a simple way by taking a detour via the Pigouvian solution described in Appendix A.1. Recall that the utilitarian objective yields precisely this solution so that $\theta^{U}=\theta^{P}$ and $s^{U}=s^{P}$. It then follows from equation (13) that $\alpha^{U}>0$ when $\theta^{P} \bar{\varphi}\left(\theta^{P}\right)>s^{P} \bar{x}\left(s^{P}\right)$ and negative otherwise. In words, $\alpha$ is positive if the proceeds from the Pigouvian fat tax exceed the budgetary cost of the Pigouvian subsidy on the healthy good.

\subsection{Rawlsian welfare function}

A Rawlsian social planner maximizes utility as given by (19) of the worst-off individual in society, namely the one with the lowest income $y_{\ell}$. The optimization problem then amounts to

$$
\max _{\theta, \alpha} W^{R}(\theta, \alpha)=V_{\ell}(\theta, \alpha)
$$

Proceeding as in the previous section, we use equations (4) and (5) to simplify the FOCs of (26) with respect to $\theta$ and $\alpha$. They can be written as

$$
\begin{aligned}
& \frac{\partial W^{R}}{\partial \theta}=-\frac{\partial \tau}{\partial \theta}-\varphi_{\ell}^{*}+\frac{\partial s}{\partial \theta} x_{\ell}^{*}+\frac{1}{\theta} \Omega_{\ell}\left(\beta_{\ell}, \delta_{\ell}\right), \\
& \frac{\partial W^{R}}{\partial \alpha}=-\frac{\partial \tau}{\partial \alpha}+\frac{\partial s}{\partial \alpha} x_{\ell}^{*}+\left(1-\delta_{\ell}\right) \frac{\partial b_{\ell}}{\partial x_{i}^{*}} \frac{\partial x_{\ell}^{*}}{\partial s} \frac{\partial s}{\partial \alpha},
\end{aligned}
$$

where

$$
\Omega_{\ell}\left(\beta_{\ell}, \delta_{\ell}\right) \equiv\left(1-\beta_{\ell}\right) \varepsilon_{h_{\ell}, \theta} h\left(\varphi_{\ell}^{*}\right)+\left(1-\delta_{\ell}\right) \varepsilon_{b_{\ell}, \theta} b\left(x_{\ell}^{*}\right)>0 .
$$


The interpretations of equations (27) and (28) are similar to those of equations (23) and (24), except that the direct utility and Pigouvian effects are now evaluated for the lowest income individual rather than averaged over the entire population. Observe that unlike in the utilitarian case we no longer get "straight" Pigouvian tax and subsidy rates. This is because we are in a second-best setting with restricted instruments. Consequently, the optimal policy is not longer determined solely by efficiency considerations; redistribution now also matters.

Setting (27) equal to zero and rearranging gives the following expression for the Rawlsian fat $\operatorname{tax} \theta^{R}$,

$$
\theta^{R}=\frac{\bar{\pi} \varepsilon_{\bar{\pi}, \theta}+\Omega\left(\beta_{\ell}, \delta_{\ell}\right)}{\bar{\varphi}\left[\frac{\varphi_{\ell}^{*}}{\bar{\varphi}}-\left(1-\varepsilon_{\bar{\varphi}, \theta}\right)\left(\alpha+(1-\alpha) \frac{1}{1+\varepsilon_{\bar{x}, s}} \frac{x_{\ell}^{*}}{\bar{x}}\right)\right]} .
$$

Since $\varphi_{\ell}^{*}>\bar{\varphi}$, we thus also get a positive fat tax in the Rawlsian case as long as $\alpha>0$. When $\alpha<0$, on the other hand a negative solution to (29) cannot be ruled out, which given our non negativity restriction on taxes and subsidies implies a corner solution at $\theta^{R}=0$. An alternative, and more intuitive perspective on this issue is provided by equation (A.8) in Appendix A.2. It shows that the regressive effect of the fat tax, as measured by the term $\left(\varphi_{\ell}^{*}-\bar{\varphi}\right)$, may outweigh the Pigouvian terms and thus yield $\theta^{R}=0$

Intuitively, since the fat tax is regressive, one might expect the Rawlsian level to be lower than the utilitarian one. However, the lowest income individual suffers most from misperception of the adverse health effects of fat consumption. Consequently, the Pigouvian term linked to misperception will be higher in the Rawlsian case. To show this formally, the comparison of (25) and (29) is not very informative because the level of $\alpha$ will differ. We can more easily compare (A.4) and (A.8), in Appendices A.1 and A.2. The denominator of these expressions and the first term in the numerator are the same. ${ }^{9}$ Consequently, we have $\theta^{R}<\theta^{U}=\theta^{P}$ if

$$
\left(1-\beta_{\ell}\right) h^{\prime}\left(\varphi_{\ell}^{*}\right)\left(-\frac{\partial \varphi_{\ell}^{*}}{\partial \theta}\right)-\int_{y_{\ell}}^{y_{h}}\left[\left(1-\beta_{i}\right) h^{\prime}\left(\varphi_{i}^{*}\right)\left(-\frac{\partial \varphi_{i}^{*}}{\partial \theta}\right)\right] \mathrm{d} F(y)<\left(\varphi_{\ell}^{*}-\bar{\varphi}\right) .
$$

In words, the Rawlsian case leads to a lower fat tax when the regressive effect, measured by the RHS of (30), is stronger than the Pigouvian tax differential associated with the misperception, measured by the LHS of (30). Recall that misperception increases with

\footnotetext{
${ }^{9}$ They are evaluated at different levels of $\theta$ but this is of no relevance. To establish the comparison we can for instance evaluate the FOC with respect to $\theta^{R}$ at $\theta^{U}$.
} 
income. Consequently, individual $\ell$ 's misperception will, roughly speaking, be larger than the average degree of misperception and this calls for a larger tax in the Rawlsian case.

The levels of the subsidy on the healthy good $s^{U}$ and $s^{R}$ can be compared along the same lines, yielding again a conflict between correction for misperception and regressive bias. This discussion also shows that even if we assume for instance $\theta^{R}<\theta^{U}$ and $s^{R}<s^{U}$; the comparison between $\alpha^{R}$ and $\alpha^{U}$ remains ambiguous. This follows from a simple inspection of equation (13).

\section{The political economy of fat taxes}

We now assume that the level of the fat tax is chosen by majority voting, for a given budgetary rule. Now, the variable $\alpha$ which was of no direct relevance in the normative case will be of crucial importance. With a continuum of individuals each voting agent has zero mass implying that an individual vote cannot change the outcome of the election. Individuals vote for the level of the fat tax that maximizes their perceived indirect utility function as given by (18): ${ }^{10}$

$$
\max _{\theta} \widehat{V}_{i}(\theta, \alpha) \quad \theta \geq 0 .
$$

The FOC of a type- $i$ agent with respect to $\theta$ is given by

$$
\begin{aligned}
\frac{\partial \widehat{V}_{i}}{\partial \theta}= & -\frac{\partial \tau}{\partial \theta}-\varphi_{i}^{*}-\left[(1+\theta)-\frac{\partial v_{i}}{\partial \varphi_{i}^{*}}+\beta_{i} \frac{\partial h_{i}}{\partial \varphi_{i}^{*}}\right] \frac{\partial \varphi_{i}^{*}}{\partial \theta} \\
& +\frac{\partial s}{\partial \theta} x_{i}^{*}-\left[(1-s)-\delta_{i} \frac{\partial b_{i}}{\partial x_{i}^{*}}\right] \frac{\partial x_{i}^{*}}{\partial s} \frac{\partial s}{\partial \theta} \leq 0 .
\end{aligned}
$$

Using the FOCs of the individual choices over $x_{i}$ and $\varphi_{i}$, equations (4) and (5), this equation reduces to

$$
-\frac{\partial \tau}{\partial \theta}-\varphi_{i}^{*}+\frac{\partial s}{\partial \theta} x_{i}^{*} \leq 0
$$

The first term represents the marginal benefit of higher fat taxes which is given by the reduction in the capitation fee. The second term reflects the individual's direct costs of fat taxes. These are higher for lower income individuals since they consume more of the fat good. The last term represents the benefit of a higher fat tax when part of

\footnotetext{
${ }^{10}$ Individuals vote in accordance with their perceived preferences which is in contrast to Haavio and Katakorpi (2011). In their framework individuals consume according to their perceived preferences, but vote according to their true preferences. The voting on the fat tax is thus used as a self-commitment device to overcome their self-control problems in the fat good consumption. See the Conclusion for further discussion.
} 
its revenue is employed to subsidize the healthy good consumption. Since low income individuals consume less of the healthy good they also profit less from its subsidization.

For preferences to be single peaked we assume that the second-order condition is always non-positive, i.e. $S O C_{\theta} \leq 0 .{ }^{11}$ To determine the median voter, we need to know how the most preferred fat tax rate changes with income. Differentiating (31) we get

$$
\frac{\partial \theta_{i}}{\partial y_{i}}=-\frac{-\frac{\partial \varphi_{i}}{\partial \beta_{i}} \frac{\partial \beta_{i}}{\partial y_{i}}+\frac{\partial s}{\partial \theta} \frac{\partial x_{i}}{\partial \delta_{i}} \frac{\partial \delta_{i}}{\partial y_{i}}}{S O C_{\theta}}>0 .
$$

That is, the most preferred fat tax rate is increasing in an individual's income. This is intuitive: higher income individuals consume less fat goods and are thus affected less by their taxation. In addition, high income agents profit more if some fat tax revenue is employed to subsidize healthy good consumption. Since $\theta$ is a monotonic increasing function of $y_{i}$, the median voter is simply the individual with median income, $y_{m}$. With single-peaked preferences, the equilibrium fat tax corresponds to the most preferred level of the median individual and is implicitly determined by

$$
-\frac{\partial \tau}{\partial \theta}-\varphi_{m}^{*}+\frac{\partial s}{\partial \theta} x_{m}^{*}=0
$$

where $\varphi_{m}^{*}$ and $x_{m}^{*}$ are the fat and healthy good consumption of the median voter.

How does the fat tax in the political equilibrium compare to the socially optimal level? For a utilitarian objective we have to evaluate the FOC of the social planner, equation (23) at $\theta_{m}$, i.e.

$$
\left.\frac{\partial \mathcal{W}^{U}}{\partial \theta}\right|_{\theta=\theta_{m}}=\underbrace{\left(\varphi_{m}^{*}-\bar{\varphi}\right)}_{>0}+\underbrace{\left(\bar{x}-x_{m}^{*}\right) \frac{\partial s}{\partial \theta}}_{>0}+\underbrace{\frac{1}{\theta_{m}} \Omega(\beta, \delta)}_{>0}>0 .
$$

Assuming that the income distribution satisfies the standard property that $y_{m}<\bar{y}$, we have $\bar{\varphi}<\varphi_{m}^{*}$ and $x_{m}^{*}<\bar{x}$ if $\varphi$ is a concave function while $x$ is a convex function of $y .{ }^{12}$

${ }^{11}$ Specifically, the second order condition is given by

$$
S O C_{\theta} \equiv \frac{\partial^{2} V_{i}}{\partial \theta^{2}}=-\frac{\partial^{2} \tau}{\partial \theta^{2}}-\frac{\partial \varphi_{i}^{*}}{\partial \theta}+\frac{\partial^{2} s}{\partial \theta^{2}} x_{i}^{*}+\frac{\partial s}{\partial \theta} \frac{\partial x_{i}^{*}}{\partial s} \frac{\partial s}{\partial \theta} \leq 0 \quad \forall i .
$$

${ }^{12}$ To see this observe that the concavity of $\varphi$, the property that $\varphi$ decreases with $y$ and $y_{m}<\bar{y}$ imply that

$$
\bar{\varphi} \equiv \int_{y_{\ell}}^{y_{h}} \varphi(\theta, \beta(y)) d F(y) \leq \varphi(\theta, \beta(\bar{y})) \leq \varphi\left(\theta, \beta\left(y_{m}\right)\right) \equiv \varphi_{m}^{*}
$$

Similarly, the convexity of $x$ and the property that $\delta$ is increasing in $y$ implies

$$
\bar{x} \equiv \int_{y_{\ell}}^{y_{h}} x(\theta, \delta(y)) d F(y) \geq x(\theta, \delta(\bar{y}))>x\left(\theta, \delta\left(y_{m}\right)\right) \equiv x_{m}^{*} .
$$


Under these assumptions, the fat tax in the political equilibrium is always lower than the social optimal fat tax, $\theta^{m}<\theta^{U}$. Intuitively, this result can be understood as follows. First, the median voter suffers more from taxation of fat goods since his consumption is above average consumption. Additionally, he profits less from the subsidization of healthy goods since his healthy good consumption is below average. Third, the median voter does not take into consideration the sum of all (negative) long-run effects of fat good consumption on society, nor the additional benefits of increased healthy good consumption when a share of the fat tax revenue is earmarked to subsidize healthy goods.

Turning to the Rawlsian case, evaluating the FOC for the maximization of welfare, equation (27), at $\theta_{m}$ yields

$$
\left.\frac{\partial \mathcal{W}^{R}}{\partial \theta}\right|_{\theta=\theta_{m}}=\underbrace{\left(\varphi_{m}^{*}-\varphi_{\ell}^{*}\right)}_{<0}+\underbrace{\left(x_{\ell}^{*}-x_{m}^{*}\right) \frac{\partial s}{\partial \theta}}_{<0}+\underbrace{\frac{1}{\theta_{m}} \Omega\left(\beta_{\ell}, \delta_{\ell}\right)}_{>0} .
$$

Since $\varphi_{m}^{*}<\varphi_{\ell}^{*}, x_{\ell}^{*}<x_{m}^{*}$, and $\partial s / \partial \theta>0$, the first two terms on the RHS are negative, whereas $\Omega_{\ell}\left(\beta_{\ell}, \delta_{\ell}\right)>0$. Consequently, the sign of (35) is ambiguous and it is not clear how the political fat tax compares to the socially optimal level. Unlike in the utilitarian case, the equilibrium tax rate can be smaller than the social optimal fat tax.

Intuitively, this ambiguity arises for two reasons. First, the worst-off individual consumes more of the fat good than the median voter and thus also suffers more from its taxation. Second, the worst-off individual has the lowest healthy good consumption and profits the least from its subsidization. At the same time, however, the worst-off individual has the highest misperception with respect to the health effects of the fat and healthy good consumption. He is thus the one who profits most from the positive health effects that come along with a lower consumption of fat goods due to their taxation. And this effect may drag the Rawlsian social optimal fat tax above the political fat tax.

So far, we have studied the voting equilibrium for a given level of $\alpha$. To study the constitutional stage we need to know how the fat tax relates to the budgetary rule. Earmarking fat tax revenue to the health care scheme or to the healthy good consumption may then open up the possibility to draw the political fat tax closer to its social optimum. The derivative of $\theta_{m}$ wrt $\alpha$ is

$$
\frac{\partial \theta_{m}}{\partial \alpha}=-\frac{-\frac{\partial^{2} \tau}{\partial \theta \partial \alpha}+\frac{\partial^{2} s}{\partial \theta \partial \alpha} x_{m}^{*}+\frac{\partial s}{\partial \theta} \frac{\partial x_{m}^{*}}{\partial s} \frac{\partial s}{\partial \alpha}}{S O C_{\theta}} .
$$


Rearranging yields

$$
\begin{aligned}
\frac{\partial \theta_{m}}{\partial \alpha} & =\frac{\bar{\pi}_{\theta \alpha}+\bar{\varphi}\left(1-\varepsilon_{\bar{\varphi}, \theta}\right)\left[\frac{x_{m}^{*}}{\bar{x}} \frac{1}{\left(1+\varepsilon_{\bar{x}, s}\right)}-1+\frac{(1-\alpha) \theta \bar{\varphi}}{\left[\bar{x}\left(1+\varepsilon_{\bar{x}, s}\right)\right]^{2}} \frac{\partial x_{m}^{*}}{\partial s}\right]}{S O C_{\theta}} \\
& =\frac{\bar{\pi}_{\theta \alpha}+\bar{\varphi}\left(1-\varepsilon_{\bar{\varphi}, \theta}\right)\left[\frac{x_{m}^{*}}{\bar{x}} \frac{1}{1+\varepsilon_{\bar{x}, s}}\left(1+\frac{\varepsilon_{x_{m}, s}}{1+\varepsilon_{\bar{x}, s}}\right)-1\right]}{S O C_{\theta}} .
\end{aligned}
$$

Assume that $\bar{\pi}_{\theta \alpha}<0$. Roughly speaking this means that healthy consumption (or physical activity) is more beneficial (at the margin) for individuals who consume more fat. In other words, a heavy consumption of fat goods makes the compensation by an otherwise healthy diet more effective. Then, a sufficient condition for (36) to be positive is that

$$
\frac{1+\varepsilon_{\bar{x}, s}+\varepsilon_{x_{m}, s}}{\left(1+\varepsilon_{\bar{x}, s}\right)^{2}}<\frac{\bar{x}}{x_{m}^{*}} .
$$

For isoelastic demand, i.e. $\varepsilon_{x_{m}, s}=\varepsilon_{\bar{x}, s}$, this condition is satisfied and we have $\partial \theta_{m} / \partial \alpha>$ 0 , a property we shall assume for the remainder of the paper.

\section{Earmarking of fat tax revenue}

So far, we have studied the voting equilibrium fat tax for a given level of $\alpha$. Together with the budget constraint this also determines $s$, the subsidy on the healthy good. We have shown that the voting equilibrium tax is always lower than the utilitarian solution, but that it may be lower or higher than the Rawlsian level. Furthermore, this tax increases with $\alpha$. We now turn to the constitutional stage at which the earmarking rule is determined by a welfare maximizing (utilitarian or Rawlsian) government anticipating the induced voting equilibrium. We study if and how the earmarking rule can be used to bring about the "best" possible political equilibrium. Intuitively, one would expect that a utilitarian government sets a higher than otherwise optimal level of $\alpha$ in order to boost the political support for the fat tax. ${ }^{13}$ However, we show that the determination of the earmarking rule is more complex than this first intuition would suggest. Specifically, the setting of the earmarking rule may involve a conflict between the fat tax and the healthy good subsidy.

\footnotetext{
${ }^{13}$ This intuition is in line with Casamatta et al. (2000) who deal with a problem of pension design. The generosity is determined by voting, but the degree to which the system redistributes within generations (the Bismarckian factor) is set at the constitutional stage. They show that it may be desirable to make the system more Bismarckian (less redistributive) in order to boost political support.
} 


\subsection{Utilitarian objective}

With a utilitarian objective, the constitutional problem consists in maximizing the sum of all utilities, evaluated at the induced voting equilibrium with respect to $\alpha$. Formally, the solution of $\alpha$ is defined as

$$
\alpha^{E U}=\arg \max _{\alpha}\left\{W^{U}(\alpha)=\int_{y_{\ell}}^{y_{h}} V_{i}\left(\theta_{m}(\alpha), \alpha\right) \mathrm{d} F(y) \quad \text { s.t. } \quad \alpha \leq 1\right\},
$$

Differentiating $W(\alpha)$ with respect to $\alpha$, we have

$$
\frac{\mathrm{d} W^{U}}{\mathrm{~d} \alpha}=\int_{y_{\ell}}^{y_{h}} \frac{\partial V_{i}}{\partial \theta_{m}} \frac{\partial \theta_{m}}{\partial \alpha} \mathrm{d} F(y)+\int_{y_{\ell}}^{y_{h}} \frac{\partial V_{i}}{\partial \alpha} \mathrm{d} F(y) .
$$

Since the political fat tax is too low for all possible $\alpha$ 's and with $\theta_{m}$ increasing in $\alpha$ one might expect that the level of $\alpha$ set at the constitutional stage would be higher than $\alpha^{U}$. In other words, we devote a higher proportion of tax proceeds to the financing of health care (the reduction of $\tau$ ) than otherwise optimal. Put differently, a smaller than optimal share would be devoted to the subsidization of the healthy good. To verify this conjecture we have to evaluate (37) at $\alpha^{U}$. We know from our earlier results that the first term on the RHS is positive and it represents the intuitive argument we just described. Boosting $\alpha$ will bring the $\theta$ supported by the median voter closer to its optimal level. In other words, $\alpha$ is used to boost political support for the fat tax. However, this intuition has to be qualified because the second term on the RHS is negative. To see this, observe that from equation (A.3) in the Appendix it follows that

$$
\int_{y_{\ell}}^{y_{h}} \frac{\partial V_{i}}{\partial \alpha} \mathrm{d} F(y)=\int_{y_{\ell}}^{y_{h}}\left[-\frac{\partial \pi_{i}}{\partial x_{i}}-s+\left(1-\delta_{i}\right) b^{\prime}\left(x_{i}\right)\right] \frac{\partial x_{i}}{\partial s} \mathrm{~d} F(y) \frac{\partial s}{\partial \alpha} .
$$

Now, we know from (16) that $\partial s / \partial \alpha<0$. The remainder of the RHS of (38) is simply $\mathrm{d} \mathcal{W}^{U} / \mathrm{d} s$, where $\mathcal{W}^{U}$ is utilitarian welfare as defined by (A.1). Now with $\alpha=\alpha^{U}$ and $\theta=\theta_{m}<\theta^{U}$ we must have from (11) that $s<s^{U}$, which from the second order condition implies that $\mathrm{d} \mathcal{W} / \mathrm{d} s>0$. Putting these elements together shows that the second term on the RHS of (37) is negative at $\alpha^{U}$. Consequently, the entire expression does not appear to have an unambiguous sign. Intuitively, this is because the increase in $\alpha$ also has a negative effect, namely that it further decreases $s$ which is already too low.

To sum up, while the voting equilibrium tax is always too low and while increasing $\alpha$ over and above the utilitarian level does boost political support for the fat tax and increase its level, it is not necessarily optimal to do so. This is because the optimal policy 
may involve a tradeoff between the (political equilibrium) fat tax and the healthy good subsidy. Increasing the share of tax proceed devoted to health insurance may further decrease the subsidy which is already too low.

\subsection{Rawlsian objective}

With a Rawlsian objective, the constitutional problem consists in maximizing the utility of the worst-off individual with respect to $\alpha$. Formally, the solution of $\alpha$ is defined as

$$
\alpha^{E R}=\arg \max _{\alpha}\left\{W^{R}=V_{\ell}\left(\theta_{m}(\alpha), \alpha\right) \text { s.t. } \alpha \leq 1\right\},
$$

Differentiating $W^{R}(\alpha)$, we get

$$
\frac{\mathrm{d} W^{R}}{\mathrm{~d} \alpha}=\frac{\partial V_{\ell}}{\partial \theta_{m}} \frac{\partial \theta_{m}}{\partial \alpha}+\frac{\partial V_{\ell}}{\partial \alpha}
$$

To study whether it is optimal to set $\alpha$ below or above the Rawlsian level obtained in the normative benchmark, we have to evaluate (39) at $\alpha^{R}$. Recall that unlike in the utilitarian case, the voting equilibrium level $\theta_{m}\left(\alpha^{R}\right)$ can now be smaller or larger than $\theta^{R}$. When $\theta_{m}\left(\alpha^{R}\right)<\theta^{R}$, the first term on the RHS of (39) is once again positive. And an argument which is exactly similar to the one used in the previous subsection (using equation (A.7) rather than (A.3) in the Appendix) can be used to show that the second term is negative. Consequently, the sign of the overall expression is ambiguous. On the other hand, when $\theta_{m}\left(\alpha^{R}\right)<\theta^{R}$, the first term on the RHS of (39) is negative. And using once again the same argument for the second term it follows that it is now positive. To sum up, while the respective signs of the two terms may be reversed, they always go in opposite directions. Intuitively, this is essentially due to a simple accounting argument, based on expression (11). Given $\alpha=\alpha^{R}$, when $\theta_{m}$ is too low, $s$ is also too low (so that the second term is negative). But when $\theta_{m}$ is too large, $s$ is also too large and the second term is positive (because an increase in $\alpha$ brings about a decrease in the level of $s$ in the voting equilibrium).

\section{Conclusion}

This paper has studied the determination of a fat tax and a subsidy on a healthy good through majority voting. The voting procedure is preceded by a constitutional stage in which a welfare maximizing (utilitarian or Rawlsian) government sets the earmarking 
rule, which specifies the share of fat tax proceeds that is used to contribute to the funding of the health care system, while the remaining revenue is used to finance the subsidy on the healthy good. Both the long-run costs of fat consumption and the long-run benefits of the healthy good are misperceived. In addition, individuals choose their consumption levels without taking into account their impact on health care costs. Voting behavior is based on the misperceived utility. In other words, the problem is not one of self-control but one of inadequate information.

We show that the equilibrium fat tax is always lower than at the utilitarian benchmark, while it may be lower or higher than the Rawlsian level. Furthermore, the equilibrium tax increases with $\alpha$, the share of tax proceeds devoted to health insurance. Roughly speaking this is the case because increasing $\alpha$ makes the overall tax policy less regressive. Indeed, a larger subsidy on the healthy good makes the policy more regressive.

In the utilitarian case we show that even though the earmarking rule can be used to boost the political support for the fat tax, it is not necessarily optimal to do so. This is because there is a conflict between the fat tax and the healthy good subsidy. The latter is also too low at the voting equilibrium and may be further decreased as $\alpha$ increases. To sum up, the level of $\alpha$ set at the constitutional stage may be lower as well as higher than the utilitarian (full control) benchmark. Similar effects are at work in the Rawlsian case with the added complexity that the voting equilibrium tax level may then actually exceed the optimal level.

To sum up, earmarking is an effective tool to influence the voting equilibrium tax/subsidy policy and bring it closer to the optimum. However, the determination of the optimal earmarking rule is more complex then on would have expected at first. It is not simply used to boost political support for the fat tax. Instead, it may involve a tradeoff between the (political equilibrium) fat tax and the healthy good subsidy. Consequently, the optimal level of $\alpha^{P}$ may actually be lower than the utilitarian benchmark $\alpha^{E U}$ in order to ensure an more adequate level of the healthy good subsidy.

We have assumed that individuals vote according to their misperceived preferences. Haavio and Kotakorpi (2011) investigate a problem of self-control over sin goods and consequently assume that the median voter votes accordingly to his long-term utility function. In our setup this translates into assuming that individuals vote based on 
preferences with $\beta=\delta=1$. In this case the median voter chooses the fat tax while being aware of his own misperception of the utility costs and benefits of fat and healthy goods. ${ }^{14}$ In other words he uses the tax policy as a commitment devise to mitigate his "self-control" problem. Consequently the first of the two externalities described in Section 3 is accounted for, at least in part. ${ }^{15}$ The second, health insurance cost, externality remains however relevant. Overall we can thus expect $\theta_{m}$ to be further away from the utilitarian choice when individuals vote according to their misperceived utility function. On that account we believe that understanding if individuals are affected by misperception - and vote accordingly to their misperceived preferences - or by temptation - and vote accordingly to the true effects - has important policy implications.

\footnotetext{
${ }^{14}$ The analogous of (33) would be

$$
-\frac{\partial \tau}{\partial \theta}-\varphi_{m}^{*}\left(1-\beta_{m}\right) \frac{\partial h_{m}}{\partial \varphi_{m}^{*}} \frac{\partial \varphi_{m}^{*}}{\partial \theta}+\frac{\partial s}{\partial \theta} x_{m}^{*}-\left(1-\delta_{m}\right) \frac{\partial b_{m}}{\partial x_{m}^{*}} \frac{\partial x_{m}^{*}}{\partial s} \frac{\partial s}{\partial \theta}=0 .
$$

It comes out that the third term in (34) would be partially corrected by the median voter's misperception terms.

${ }^{15}$ To be more precise the commitment benefit is mitigated by the tax revenue effect which arises because the median voter does not get a full refund of his tax payments.
} 


\section{References}

[1] Abegunde, D., Stanciole, A., "An Estimation of the Economic Impact of Chronic Non-Communicable Disease in Selected Countries," World Health Organization Department of Chronic Diseases and Health Promotion (CHP), 2006, http://www.who.int/chp

[2] Allais, O., Bertail, P. and Nichèle, V., "The Effects of a Fat Tax on French Households' Purchases: A Nutritional Approach "American Journal of Agricultural Economics, 2010, 92 (1), 228-245.

[3] Beatty,T., and LaFrance, J., "United States Demand for Food and Nutrition in the Twentieth Century, "American Journal of Agricultural Economics, 2005, 87 (11), 59-66.

[4] Bauman, A., Reis R., Sallis, J., Wells, J., Loos R. and Martin, B., "Correlates of physical activity: why are some people physically active and others not?," The Lancet, 2012, 380, 258-271.

[5] Casamatta, G., Cremer, H. and Pestieau, P. "Political Sustainability and the Design of Social Insurance," Journal of Public Economics, 2000, 75, 341-364.

[6] Cawley, J., Meyerhoefer, C., "The Medical Care Costs of Obesity: An Instrumental Variables Approach," Journal of Health Economics, 2012, 31, 219-230.

[7] Cremer, H., De Donder, P., Maldonado, D., Pestieau, P., "Taxing Sin Goods and Subsidizing Health Care," The Scandinavian Journal of Economics, 2003, 114 (1), 101-123.

[8] Cutler, D.M., Glaeser, E.L., Shapiro, J.M., "Why American Become More Obese?," Journal of Economic Perspectives, 2003, 17 (3), 93-118.

[9] Devaux, M., Sassi, F., Church, J., Cecchini, M., Borgonovi, F., "Exploring the Relationship Between Education and Obesity," OECD Journal: Economic Studies, 2011, 2011/1, 121-159.

[10] Finkelstein, E.A., Fiebelkorn, I.C., Wang, G., "National Medical Expenditures Attributable to Overweight and Obesity: How Much, and Who is Paying?," Health Affairs, 2003, W3, 219-226. 
[11] Finkelstein, E., Yang, H.K., "Obesity and Medical Costs, The Oxford Handbook of the Social Science of Obesity,", Ed. John Cawley, New York: Oxford University Press, 2011. 496-501.

[12] Finkelstein, E. A., Trogdon, J. G., Brown, D. S., Allaire, B. T., Dellea, P. S. and Kamal-Bahl, S. J., "The Lifetime Medical Cost Burden of Overweight and Obesity: Implications for Obesity Prevention," Obesity, 2008, 16, 1843-1848.

[13] Finkelstein, E.A., Trogdon, J.G., Cohen, J.W., Dietz, W., "Annual Medical Spending Attributable to Obesity: Payer and Service-Specific Estimates," Health Affairs, 2009, w822-w831.

[14] Goulão, C., Pérez-Barahona, A., "Intergenerational Transmission of NonCommunicable Chronic Diseases", Journal of Public Economic Theory, 2014, 16 (3), 467-490.

[15] Grimes, C., Riddell, L., Nowson, C., "Consumer Knowledge and Attitudes to Salt Intake and Labelled Salt Information," Appetite, 2009, 53, 189-194.

[16] Haavio, M., Kotakorpi, K., "The Political Economy of Sin Taxes," European Economic Review, 2011, 55 (4), 575-594.

[17] Harding, M., Lovenheim, M., "The Effect of Prices on Nutrition: Comparing the Impact of Product- and Nutrient-Specific Taxes," NBER Working Paper No. 19781, January 2014.

[18] Huang, K., and Lin, B., "Estimation of Food Demand and Nutrient Elasticities from Household Survey Data,"USDA, ERS, 2000, Technical Bulletin No. 1887.

[19] Katakorpi, K., "The Incidence of Sin Taxes," Economics Letters, 2008, 98, 95-99.

[20] Leicester, A., and Windmeijer, F., (2004) "The 'Fat Tax': Economic Incentives to Reduce Obesity, "IFS Briefing Notes BN49, Institute for Fiscal Studies: London, UK.

[21] McLaren, Lindsay, "Socioeconomic Status and Obesity,", Ed. John Cawley, New York: Oxford University Press, 2011. 276-288. 
[22] O'Donoghue, T., Rabin, M., "Studying Optimal Paternalism, Illustrated by a Model of Sin Taxes" American Economic Review: Papers and Proceedings, 2003, 92 (2), 186-191.

[23] O'Donoghue, T., Rabin, M., "Optimal Sin Taxes," Journal of Public Economics, 2006, 90, 1825-1849.

[24] Pollard, C., Miller, M., Woodman, R., Meng, R., Binns, C., "Changes in Knowledge, Beliefs, and Behavior Related to Fruit and Vegetable Consumption Among Western Australian Adults from 1995 to 2004," American Journal of Public Health, 2009, 99 (2), 355-361.

[25] Powell, L. M., Chaloupka, F. J., "Food Prices and Obesity: Evidence and Policy Implications for Taxes and Subsidies," Milbank Quarterly, 2009, 87 (1), 229257.

[26] Schwartz, J., Byrd-Bredbenner, C., "Young Adults' Knowledge of Standard Serving Sizes and Their Ability to Estimate Servings in Typical Portions," Journal of the American Dietetic Association, 2005, 105 (8), Supplement, 59-59.

Spasojevic 2003

[27] Thorpe, K.E., Curtis, S.F., Howard, D.H., Joski, P.., "Trends: The Impact of Obesity on Rising Medical Spending," Health Affairs, 2004, W4, 480-486.

[28] Vereecken, C., Maes, L., "Young Children's Dietary Habits and Associations with the Mother's Nutritional Knowledge and Attitudes," Appetite, 2010, 54, 44-51.

[29] Yaniv, G., Rosin, O., Tobol, Y., "Junk-Food, Home Cooking, Physical Activity and Obesity: The effect of the Fat Tax and the Thin Subsidy," Journal of Public Economics, 2009, 93, 823-830.

[30] Wang, Y., Beydoun, M.A., Liang, L., Caballero, B., Kumanyika, "Will All Americans Become Overweight or Obese? Estimating the Progression and Cost of the US Obesity Epidemic," Obesity, 2008, 16(10), 2323-2330.

[31] Wang, Y.C., McPherson, K., Marsh, T., Gortmaker, S.L., Brown, M., "Health and Economic Burden of the Projected obesity Trends in the USA and the UK," The Lancet, 2011, 378, 815-825. 
[32] WHO Global Infobase, https://apps.who.int/infobase/comparestart.aspx, accessed on November 1, 2009.

\section{A Appendix}

\section{A.1 Pigouvian taxes and subsidies}

To obtain the Pigouvian levels $\theta^{P}$ and $s^{P}$, we solve

$$
\begin{array}{rl}
\max _{\theta, \tau, s} & \mathcal{W}(\theta, \tau, s)=\int_{y_{\ell}}^{y_{h}}\left[y_{i}-\tau-(1+\theta) \varphi_{i}^{*}-(1-s) x_{i}^{*}+b\left(x_{i}^{*}\right)+v\left(\varphi_{i}^{*}\right)-h\left(\varphi_{i}^{*}\right)\right] \mathrm{d} F(y) . \\
\text { s.t. } & \tau+\theta \bar{\varphi}(\theta)=\bar{\pi}(\theta, s)+s \bar{x}(s)
\end{array}
$$

substituting from the budget constraint and adding and subtracting $\delta_{i} b\left(x_{i}^{*}\right)-\beta_{i} h\left(\varphi_{i}^{*}\right)$, $\mathcal{W}$ can be rewritten as

$$
\begin{aligned}
\mathcal{W} & =\int_{y_{\ell}}^{y_{h}}\left[y_{i}-\bar{\pi}(\theta, s)-s \bar{x}(s)+\theta \bar{\varphi}(\theta)-(1+\theta) \varphi_{i}^{*}-(1-s) x_{i}^{*}+\delta_{i} b\left(x_{i}^{*}\right)+v\left(\varphi_{i}^{*}\right)\right. \\
& \left.-\beta_{i} h\left(\varphi_{i}^{*}\right)+\left(1-\delta_{i}\right) b\left(x_{i}^{*}\right)-\left(1-\beta_{i}\right) h\left(\varphi_{i}^{*}\right)\right] \mathrm{d} F(y)
\end{aligned}
$$

Differentiating and rearranging by making use of the envelope theorem yields

$$
\begin{gathered}
\int_{y_{\ell}}^{y_{h}}\left[-\frac{\partial \pi_{i}}{\partial \varphi_{i}^{*}}+\theta-\left(1-\beta_{i}\right) h^{\prime}\left(\varphi_{i}^{*}\right)\right] \frac{\partial \varphi_{i}^{*}}{\partial \theta} \mathrm{d} F(y)=0, \\
\int_{y_{\ell}}^{y_{h}}\left[-\frac{\partial \pi_{i}}{\partial x_{i}^{*}}-s+\left(1-\delta_{i}\right) h^{\prime}\left(x_{i}^{*}\right)\right] \frac{\partial x_{i}^{*}}{\partial s} \mathrm{~d} F(y)=0
\end{gathered}
$$

rearranging and "solving" yields

$$
\begin{aligned}
& \theta^{P}=\frac{\int_{y^{\ell}}^{y_{h}}\left[\frac{\partial \pi_{i}}{\partial \varphi_{i}^{*}}+\left(1-\beta_{i}\right) h^{\prime}\left(\varphi_{i}^{*}\right)\right]\left(-\frac{\partial \varphi_{i}^{*}}{\partial \theta}\right) \mathrm{d} F(y)}{-\frac{\partial \bar{\varphi}}{\partial \theta}}>0, \\
& s^{P} \quad=\frac{\int_{y_{\ell}}^{y_{h}}\left[-\frac{\partial \pi_{i}^{*}}{\partial x_{i}^{*}}+\left(1-\delta_{i}\right) b^{\prime}\left(x_{i}^{*}\right)\right] \frac{\partial x_{i}^{*}}{\partial s} \mathrm{~d} F(y)}{\frac{\partial \bar{x}}{\partial s}}>0 .
\end{aligned}
$$

\section{A.2 Rawlsian taxes and subsidies}

To obtain the Rawlsian solution by using taxes and subsidies as decision variables we determine $\theta^{R}$ and $s^{R}$, by

$$
\begin{aligned}
\max _{\theta, \tau, s} & \mathcal{W}^{R}(\theta, \tau, s)=\left[y_{\ell}-\tau-(1+\theta) \varphi_{\ell}^{*}-(1-s) x_{\ell}^{*}+b\left(x_{\ell}^{*}\right)+v\left(\varphi_{\ell}^{*}\right)-h\left(\varphi_{\ell}^{*}\right)\right] \\
\text { s.t. } & \tau+\theta \bar{\varphi}(\theta)=\bar{\pi}(\theta, s)+s \bar{x}(s)
\end{aligned}
$$


substituting from the budget constraint and adding and subtracting $\delta_{\ell} b\left(x_{\ell}^{*}\right)-\beta_{\ell} h\left(\varphi_{\ell}^{*}\right)$, $\mathcal{W}^{R}$ can be rewritten as

$$
\begin{aligned}
\mathcal{W}= & y_{\ell}^{*}-\bar{\pi}(\theta, s)-s \bar{x}(s)+\theta \bar{\varphi}(\theta)-(1+\theta) \varphi_{\ell}^{*}-(1-s) x_{\ell}^{*}+\delta_{\ell} b\left(x_{\ell}^{*}\right)+v\left(\varphi_{\ell}^{*}\right)-\beta_{\ell} h\left(\varphi_{\ell}^{*}\right) \\
& +\left(1-\delta_{\ell}\right) b\left(x_{\ell}^{*}\right)-\left(1-\beta_{\ell}\right) h\left(\varphi_{\ell}^{*}\right) .
\end{aligned}
$$

Differentiating and rearranging, by making use of the envelope theorem, yields

$$
\begin{aligned}
\int_{y_{\ell}}^{y_{h}}\left[-\frac{\partial \pi_{i}}{\partial \varphi_{i}^{*}}+\theta\right] \frac{\partial \varphi_{i}^{*}}{\partial \theta} \mathrm{d} F(y)+\left(1-\beta_{\ell}\right) h^{\prime}\left(\varphi_{\ell}^{*}\right)+\left(\bar{\varphi}-\varphi_{\ell}^{*}\right) & =0 \\
\int_{y_{\ell}}^{y_{h}}\left[-\frac{\partial \pi_{i}}{\partial x_{i}^{*}}-s\right] \frac{\partial x_{i}^{*}}{\partial s} \mathrm{~d} F(y)+\left(1-\delta_{\ell}\right) b^{\prime}\left(x_{\ell}^{*}\right)+\left(x_{\ell}^{*}-\bar{x}\right) & =0
\end{aligned}
$$

rearranging and "solving" yields

$$
\begin{aligned}
\theta^{R} & =\frac{\int_{y_{\ell}}^{y_{h}} \frac{\partial \pi_{i}}{\partial \varphi_{i}^{*}}\left(-\frac{\partial \varphi_{i}^{*}}{\partial \theta}\right) \mathrm{d} F(y)+\left(1-\beta_{\ell}\right) h^{\prime}\left(\varphi_{\ell}^{*}\right)\left(-\frac{\partial \varphi_{\ell}^{*}}{\partial \theta}\right)-\left(\varphi_{\ell}^{*}-\bar{\varphi}\right)}{-\frac{\partial \bar{\varphi}}{\partial \theta}}, \\
s^{R} & =\frac{\int_{y_{\ell}}^{y_{h}}-\frac{\partial \pi_{i}}{\partial x_{i}^{*}} \frac{\partial x_{i}^{*}}{\partial s} \mathrm{~d} F(y)+\left(1-\delta_{\ell}\right) b^{\prime}\left(x_{\ell}^{*}\right) \frac{\partial x_{\ell}^{*}}{\partial s}-\left(\bar{x}-x_{\ell}^{*}\right)}{\frac{\partial \bar{x}}{\partial s}} .
\end{aligned}
$$

The denominators of both of these expressions are positive. The first two terms in the numerator of (A.8) are positive Pigouvian terms which correct for the two externalities that are present in our setting. The first measures the externality via health care costs and the second the correction for misperception. The third term, however, is negative and measures the regressive effect of the Pigouvian tax. The poorest individual pays $\theta \varphi_{\ell}^{*}$ but his refund via the reduction in $\tau$ is only $\theta \bar{\varphi}$. The numerator of (A.9) has a similar interpretation with two Pigouvian and a third redistributive term, which once again is negative. 